\title{
UNAMBIGUOUS ERASING MORPHISMS IN FREE MONOIDS*
}

\author{
Johannes C. SCHNEIDER ${ }^{1}$
}

\begin{abstract}
This paper discusses the fundamental combinatorial question of whether or not, for a given string $\alpha$, there exists a morphism $\sigma$ such that $\sigma$ is unambiguous with respect to $\alpha$, i.e. there exists no other morphism $\tau$ satisfying $\tau(\alpha)=\sigma(\alpha)$. While Freydenberger et al. [Int. J. Found. Comput. Sci. 17 (2006) 601-628] characterise those strings for which there exists an unambiguous nonerasing morphism $\sigma$, little is known about the unambiguity of erasing morphisms, i.e. morphisms that map symbols onto the empty string. The present paper demonstrates that, in contrast to the main result by Freydenberger et al., the existence of an unambiguous erasing morphism for a given string can essentially depend on the size of the target alphabet of the morphism. In addition to this, those strings for which there exists an erasing morphism over an infinite target alphabet are characterised, complexity issues are discussed and some sufficient conditions for the (non-)existence of unambiguous erasing morphisms are given.
\end{abstract}

Mathematics Subject Classification. 68R15, 68Q25.

\section{INTRODUCTION}

The examination of the unambiguity of morphisms arises from the research on pattern languages. In contrast to the rather common setting of dealing with one single morphism applied to all strings in a set, a pattern language is the set of all morphic images of one fixed string - then called pattern (for more information on pattern languages see, e.g., Mateescu and Salomaa [8]). In the field of pattern languages, we might be confronted with the situation that two different morphisms,

\footnotetext{
Keywords and phrases. Combinatorics on words, morphisms in free monoids, unambiguity, complexity.

* A preliminary version of this paper was presented at the conference SOFSEM 2009 (cf. [12]).

1 Fachbereich Informatik, Technische Universität Kaiserslautern, Postfach 3049,

67653 Kaiserslautern, Germany; jschneider@informatik.uni-kl.de
} 
applied to a given pattern, generate the same morphic image. If, for a given pattern and a morphism $h$, there exists no other morphism that, applied to the pattern, generates the same image, we call this morphism $h$ unambiguous, otherwise, it is called ambiguous. Ambiguity of morphisms has great impact on the learnability of pattern languages ( $c f$. Reidenbach $[9,10])$.

The explicit detailed examination of the unambiguity of morphisms, as initiated by Freydenberger et al. [4] and continued by Freydenberger and Reidenbach [3], offers a combinatorially rich theory and shows various cross-references to other topics in combinatorics on words, such as fixed points of morphisms, equality sets and the Post correspondence problem. This paper takes up some of the open problems in Freydenberger et al. [4] - first of all the question, for which string, there exists an unambiguous erasing morphism. Some minor, preliminary results concerning this question have already been given in [4].

We now introduce the definition of unambiguous morphisms a little more formally: given an arbitrary alphabet $\Sigma$ and a string ${ }^{1} \alpha \in \mathbb{N}^{+}$, a morphism $\sigma: \mathbb{N}^{*} \rightarrow$ $\Sigma^{*}$ is called unambiguous with respect to $\alpha$ if and only if there exists no other morphism $\tau: \mathbb{N}^{*} \rightarrow \Sigma^{*}$ (i.e. $\tau(i) \neq \sigma(i)$ for a symbol $i$ occurring in $\alpha$ ) such that $\tau(\alpha)=\sigma(\alpha)$. If such a morphism $\tau$ exists, $\sigma$ is called ambiguous with respect to $\alpha$. For instance, the morphism $\sigma_{1}: \mathbb{N}^{*} \rightarrow\{\mathrm{a}, \mathrm{b}\}^{*}, \sigma_{1}(i):=\mathrm{ab}^{i}$ for every $i \in \mathbb{N}$, is ambiguous with respect to $\alpha_{1}:=1 \cdot 2 \cdot 2 \cdot 3 \cdot 1 \cdot 3$ since there exists another morphism $\tau: \mathbb{N}^{*} \rightarrow\{\mathrm{a}, \mathrm{b}\}^{*}$, given by $\tau(1):=\mathrm{a}, \tau(2):=$ bab, $\tau(3):=$ babbb, satisfying $\tau\left(\alpha_{1}\right)=\sigma_{1}\left(\alpha_{1}\right)$ :

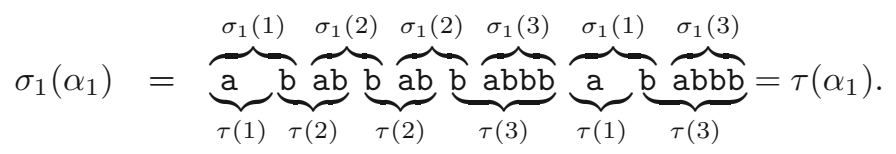

In contrast to this, every morphism $\sigma: \mathbb{N}^{*} \rightarrow \Sigma^{*}$ is unambiguous with respect to, e.g., $\alpha_{2}:=1 \cdot 1$.

In Freydenberger et al. [4], those strings $\alpha$ are characterised for which there exists an unambiguous nonerasing morphism $\sigma: \mathbb{N}^{*} \rightarrow \Sigma^{*}$, i.e. $|\sigma(i)| \geq 1$ for every symbol $i$ occurring in $\alpha$. However, there are strings for which every nonerasing morphism is ambiguous, but there exists an unambiguous erasing morphism, i.e. a morphism that maps certain symbols of the string onto the empty string $\varepsilon$. For instance, consider $\alpha_{3}:=1 \cdot 2 \cdot 2$. Every nonerasing morphism $\sigma_{\mathrm{NE}}: \mathbb{N}^{*} \rightarrow$ $\Sigma^{*}$ is ambiguous since there exists another morphism $\tau: \mathbb{N}^{*} \rightarrow \Sigma^{*}$, defined by $\tau(1):=\sigma_{\mathrm{NE}}(1 \cdot 2 \cdot 2), \tau(2):=\varepsilon$, satisfying $\sigma_{\mathrm{NE}}\left(\alpha_{3}\right)=\tau\left(\alpha_{3}\right)$. In contrast to this, every erasing morphism $\sigma_{\mathrm{E}}: \mathbb{N}^{*} \rightarrow \Sigma^{*}$ with $\left|\sigma_{\mathrm{E}}(1)\right|=1$ and $\sigma_{\mathrm{E}}(2)=\varepsilon$ is unambiguous with respect to $\alpha_{3}$. Finally, there also exist strings for which every (erasing or nonerasing) morphism is ambiguous, provided that this morphism does not map all symbols in the pattern to the empty word. This is illustrated by, e.g., $\alpha_{4}:=1 \cdot 2 \cdot 1 \cdot 2$. Let $\sigma: \mathbb{N}^{*} \rightarrow \Sigma^{*}$ be an arbitrary morphism. If $\sigma(1) \neq \varepsilon$, then the

\footnotetext{
${ }^{1}$ For the sake of convenience, we use $\mathbb{N}$ as an infinite alphabet and consider strings over $\mathbb{N}$ such as the string $2 \cdot 1 \cdot 2 \cdot 25 \cdot 17$, where $\cdot$ refers to the concatenation and is used to separate between different symbols in the string.
} 
morphism $\tau(1):=\varepsilon, \tau(2):=\sigma(1 \cdot 2)$, satisfies $\tau\left(\alpha_{4}\right)=\sigma\left(\alpha_{4}\right)$ - the case $\sigma(2) \neq \varepsilon$ is analogous. Hence, no such morphism $\sigma$ is unambiguous with respect to $\alpha_{4}$.

The main question investigated in this work is: for which strings $\alpha \in \mathbb{N}^{+}$does there exist an unambiguous morphism $\sigma: \mathbb{N}^{*} \rightarrow \Sigma^{*}$ ? In contrast to Freydenberger et al. [4], where this question is discussed primarily focussing on injective and, hence, nonerasing morphisms, we explicitly study those morphisms that "erase" symbols in $\alpha$, i.e. map symbols onto the empty word. It turns out that the answer to this question depends on the size of $\Sigma$, which establishes a new and unexpected aspect in the research on the ambiguity of morphisms since, concerning nonerasing morphisms, the size of $\Sigma$ does not matter as long as $|\Sigma| \geq 2$ (Freydenberger et al. [4]). Additionally, we characterise those strings $\alpha \in \mathbb{N}^{*}$ for which there exists an unambiguous morphism with an infinite target alphabet, discuss the complexity of the respective decision problem and, finally, give a sufficient condition for the existence of an unambiguous morphism $\sigma: \mathbb{N}^{*} \rightarrow \Sigma^{*}$ with a finite alphabet $\Sigma$.

\section{Definitions AND BASIC RESUlts}

In the present section we establish some basic definitions and notation. Parts of the terminology are adapted from the research on pattern languages ( $c f$. Mateescu and Salomaa [8]). Additionally, for notation not explained explicitly, we refer the reader to Choffrut and Karhumäki [1]. Concepts concerning decidability and complexity are adopted from Garey and Johnson [5].

Let $\mathbb{N}:=\{1,2, \ldots\}$ be the set of natural numbers. Let $M, M_{1}, M_{2}, \ldots, M_{n}$ be sets, $n \in \mathbb{N}$. $\left(M_{1}, M_{2}, \ldots, M_{n}\right)$ is called a partition of $M$ if and only if $M_{1} \cup M_{2} \cup$ $\ldots \cup M_{n}=M$ and $M_{i} \cap M_{j}=\emptyset$ for all $1 \leq i<j \leq n$.

An alphabet $\mathcal{A}$ is an enumerable set of symbols. A string (over $\mathcal{A}$ ) is a finite sequence of symbols taken from $\mathcal{A}$. Using $|X|$ we denote the cardinality of a set $X$ or the length of a string $X$. The empty string is the unique sequence of symbols of length 0 ; we use $\varepsilon$ for the empty string. For the concatenation of strings $s, t$ we write $s \cdot t$ (or $s t$ for short). The string that results from the $n$-fold concatenation of a string $s$ is denoted by $s^{n}$. The notation $\mathcal{A}^{*}$ refers to the set of all strings over $\mathcal{A}$, i.e., more precisely, the free monoid generated by $\mathcal{A}$; furthermore, $\mathcal{A}^{+}:=\mathcal{A}^{*} \backslash\{\varepsilon\}$. The number of occurrences of a symbol $x \in \mathcal{A}$ in a string $s \in \mathcal{A}^{*}$ is written as $|s|_{x}$. With regard to arbitrary strings $s, t \in \mathcal{A}^{*}$, we write $s=\ldots t$ if there exists an $u \in \mathcal{A}^{*}$ such that $s=u t$, we write $s=t \ldots$ if there exists an $u \in \mathcal{A}^{*}$ such that $s=t u$, and, finally, $s=\ldots t \ldots$ if there exist $u, v \in \mathcal{A}^{*}$ such that $s=u t v$. In contrast to this, if we wish to omit some parts of a canonically given string then we henceforth use the symbol $[\ldots]$. E.g. $\alpha=\ldots 1 \cdot 2 \cdot[\ldots] \cdot 5$ means that $\alpha$ ends with the string $1 \cdot 2 \cdot 3 \cdot 4 \cdot 5$.

We often use $\mathbb{N}$ as an infinite alphabet. In order to distinguish between strings over $\mathbb{N}$ and strings over a (possibly finite) alphabet $\Sigma$, we call the former patterns. We name patterns using lower case letters from the beginning of the Greek alphabet such as $\alpha, \beta, \gamma$. Given a pattern $\alpha \in \mathbb{N}^{*}$, we call symbols occurring in $\alpha$ variables and denote the set of variables in $\alpha$ with $\operatorname{var}(\alpha)$. Hence, $\operatorname{var}(\alpha) \subseteq \mathbb{N}$. 
Considering strings over $\mathbb{N}$, we use $\cdot$ to separate symbols in $\mathbb{N}$ in order to distinguish between, for instance, the strings $1 \cdot 1 \cdot 2$ and $11 \cdot 2$.

Given arbitrary alphabets $\mathcal{A}, \mathcal{B}$, a morphism is a mapping $h: \mathcal{A}^{*} \rightarrow \mathcal{B}^{*}$ that is compatible with the concatenation, i.e., for all $v, w \in \mathcal{A}^{*}, h(v w)=h(v) h(w)$. Hence, $h$ is fully defined for all $v \in \mathcal{A}^{*}$ as soon as it is defined for all symbols in $\mathcal{A}$. We call $h$ erasing if and only if $h(a)=\varepsilon$ for an $a \in \mathcal{A}$, otherwise $h$ is called nonerasing. If we call a morphism $h$ (non)erasing with a certain string $s$ in mind, we only demand $h$ to be (non)erasing for the symbols of $s$.

A morphism $h: \mathbb{N}^{*} \rightarrow \mathbb{N}^{*}$ is said to be nontrivial if $h(x) \neq x$ for an $x \in \mathbb{N}$. Let $V \subseteq \mathbb{N}$. We call $h: \mathbb{N}^{*} \rightarrow \mathbb{N}^{*}$ nontrivial for $V$ if $h(x) \neq x$ for an $x \in V$. We define a morphism $\pi_{V}: \mathbb{N}^{*} \rightarrow \mathbb{N}^{*}$ by $\pi_{V}(x):=x$ if $x \in V$ and $\pi_{V}(x):=\varepsilon$ if $x \notin V$. Hence, $\pi_{V}$ is the projection of a string onto its substring consisting only of symbols in $V$. Moreover, a pattern $\alpha \in \mathbb{N}^{+}$is called a fixed point of $h$ if $h(\alpha)=\alpha$. We write $g \circ h$ for the composition of arbitrary morphisms $g, h: \mathbb{N}^{*} \rightarrow \mathbb{N}^{*}$. Hence, $g \circ h(w)=g(h(w))$ for every $w \in \mathbb{N}^{*}$.

For any alphabet $\Sigma$, for any morphism $\sigma: \mathbb{N}^{*} \rightarrow \Sigma^{*}$ and for any pattern $\alpha \in \mathbb{N}^{+}$with $\sigma(\alpha) \neq \varepsilon$, we call $\sigma$ unambiguous (with respect to $\alpha$ ) if and only if there is no morphism $\tau: \mathbb{N}^{*} \rightarrow \Sigma^{*}$ satisfying $\tau(\alpha)=\sigma(\alpha)$ and, for some $x \in \operatorname{var}(\alpha), \tau(x) \neq \sigma(x)$. If $\sigma$ is not unambiguous with respect to $\alpha$, it is called ambiguous (with respect to $\alpha$ ). Furthermore, we define $\mathrm{AMB}_{\Sigma}:=\left\{\alpha \in \mathbb{N}^{+} \mid\right.$ there is no unambiguous morphism $\sigma: \mathbb{N}^{*} \rightarrow \Sigma^{*}$ with respect to $\left.\alpha\right\}$.

We conclude the definitions in this section with a partition of the set of all patterns subject to the following criterion.

Definition 2.1. Let $\alpha \in \mathbb{N}^{+}$. We call $\alpha$ prolix if and only if there exists a factorisation $\alpha=\beta_{0} \gamma_{1} \beta_{1} \gamma_{2} \beta_{2} \ldots \gamma_{n} \beta_{n}$ with $n \geq 1, \beta_{i} \in \mathbb{N}^{*}, 0 \leq i \leq n$, and $\gamma_{i} \in \mathbb{N}^{+}, 1 \leq i \leq n$, such that

(1) for every $i \in\{1,2, \ldots, n\},\left|\gamma_{i}\right| \geq 2$;

(2) for every $i \in\{0,1, \ldots, n\}$ and for every $j \in\{1,2, \ldots, n\}, \operatorname{var}\left(\beta_{i}\right) \cap \operatorname{var}\left(\gamma_{j}\right)$ $=\emptyset$

(3) for every $i \in\{1,2, \ldots, n\}$, there exists an $y_{i} \in \operatorname{var}\left(\gamma_{i}\right)$ such that $y_{i}$ occurs exactly once in $\gamma_{i}$ and, for every $i^{\prime} \in\{1,2, \ldots, n\}$, if $y_{i} \in \gamma_{i^{\prime}}$ then $\gamma_{i}=\gamma_{i^{\prime}}$.

We call $\alpha \in \mathbb{N}^{+}$succinct if and only if it is not prolix.

In the field of erasing pattern languages, a succinct pattern is the shortest generator of its respective pattern language. Furthermore, the set of prolix patterns exactly corresponds to the class of finite fixed points of morphisms:

Theorem 2.2 (Head [6]). Let $\alpha \in \mathbb{N}^{+} . \alpha$ is prolix if and only if $\alpha$ is a fixed point of a nontrivial morphism $h: \mathbb{N}^{*} \rightarrow \mathbb{N}^{*}$.

Hence, for every prolix pattern $\alpha$, there exists a morphism $h: \mathbb{N}^{*} \rightarrow \mathbb{N}^{*}$ satisfying $h(\alpha)=\alpha$ and $h(x) \neq x$ for an $x \in \operatorname{var}(\alpha)$. Note that the set of succinct patterns is also equivalent to the set of morphically primitive words (as introduced by Reidenbach and Schneider [11]). 
Regarding the unambiguity of nonerasing morphisms, the classification of patterns into succinct and prolix patterns is crucial:

Theorem 2.3 (Freydenberger et al. [4]). Let $\alpha \in \mathbb{N}^{*}$, let $\Sigma$ be an alphabet, $|\Sigma| \geq 2$. There exists an unambiguous nonerasing morphism $\sigma: \mathbb{N}^{*} \rightarrow \Sigma^{*}$ with respect to $\alpha$ if and only if $\alpha$ is succinct.

According to this result, for any prolix pattern $\alpha$, every nonerasing morphism is ambiguous. However, if we allow $\sigma$ to be erasing, $\sigma$ can still be unambiguous with respect to prolix patterns, although there are other prolix patterns, for which no morphism is unambiguous. This is demonstrated with help of the example patterns $\alpha_{3}, \alpha_{4}$ from Section 1. In the next section we examine this phenomenon in detail and, thus, primarily concentrate on prolix patterns and the question for which of those patterns there exists an unambiguous morphism.

\section{Ambiguity partitions}

We begin this section with another example pattern: let $\alpha_{5}:=1 \cdot 2 \cdot 1 \cdot 2 \cdot 3 \cdot 3 \cdot 4 \cdot 4$. $4 \cdot 4 \cdot 5 \cdot 5 \cdot 5$. This pattern is prolix since there exists a factorisation $\alpha_{5}=\beta_{0} \gamma_{1} \beta_{1} \gamma_{2} \beta_{2}$ with $\beta_{0}:=\beta_{1}:=\varepsilon, \gamma_{1}:=\gamma_{2}:=1 \cdot 2$ and $\beta_{2}:=3 \cdot 3 \cdot 4 \cdot 4 \cdot 4 \cdot 4 \cdot 5 \cdot 5 \cdot 5$, which satisfies the conditions of Definition 2.1. Hence, we can conclude from Theorem 2.3 that every morphism which is unambiguous with respect to $\alpha_{5}$ must be erasing. Indeed, we can use the same argumentation as for the example pattern $\alpha_{4}$ from Section 1 to show that a possible unambiguous morphism $\sigma: \mathbb{N}^{*} \rightarrow \Sigma^{*}$ must satisfy $\sigma(1)=\sigma(2)=\varepsilon$. Moreover, we show that $\sigma$ must also map 3 onto the empty word and argue by contradiction: assume that $\sigma(3) \neq \varepsilon$. Then $\tau: \mathbb{N}^{*} \rightarrow \Sigma^{*}$, defined by $\tau(1):=\sigma(3), \tau(2):=\tau(3):=\varepsilon, \tau(4):=\sigma(4), \tau(5):=\sigma(5)$, satisfies $\tau\left(\alpha_{5}\right)=\sigma\left(\alpha_{5}\right)$, which contradicts $\sigma$ being unambiguous. With an analogous argument, we can show that $\sigma(4)=\varepsilon$, too (with $\tau(1):=\sigma(4 \cdot 4)$ ). Thus, only variable 5 may be mapped onto a nonempty word. In fact, every morphism $\sigma: \mathbb{N}^{*} \rightarrow \Sigma^{*}$ with $|\sigma(5)|=1$ and $\sigma(x)=\varepsilon, x \neq 5$, is unambiguous with respect to $\alpha_{5}$. Informally speaking, regarding pattern $\alpha_{5}$, the need to map 1 and 2 onto the empty word provokes a type of "domino effect", which is carried forward onto 3 and 4 . To formalise this observation, we need the following definition.

Definition 3.1. Let $\alpha \in \mathbb{N}^{+}$. We inductively define an ambiguity partition (with respect to $\alpha)$ :

(i) $(\emptyset, \operatorname{var}(\alpha))$ is an ambiguity partition with respect to $\alpha$.

(ii) If $(E, N)$ is an ambiguity partition with respect to $\alpha$ and there exists a morphism $h: \mathbb{N}^{*} \rightarrow \mathbb{N}^{*}$ that is nontrivial for $N$ and satisfies $h(\alpha)=\pi_{N}(\alpha)$ then $\left(E^{\prime}, N^{\prime}\right)$ is an ambiguity partition with

$$
\begin{aligned}
E^{\prime} & :=E \cup\{x \in N \mid h(x)=\varepsilon\} \\
N^{\prime} & :=\{x \in N \mid h(x) \neq \varepsilon\} .
\end{aligned}
$$


Note that $E$ contains those variables for which it is certain that an unambiguous morphism $\sigma: \mathbb{N}^{*} \rightarrow \Sigma^{*}$ needs to map them to the empty word, whereas $N$ consists of those variables that can either be mapped by $\sigma$ to a nonempty word or for which this aspect is uncertain. This is formally shown below.

We now demonstrate how Definition 3.1 affects the above example pattern $\alpha_{5}$ : let $E:=\emptyset, N:=\operatorname{var}(\alpha)$. According to $(\mathrm{i}),(E, N)$ is an ambiguity partition of $\alpha_{5}$. Let $h: \mathbb{N}^{*} \rightarrow \mathbb{N}^{*}$ be defined by $h(1):=\varepsilon, h(2):=1 \cdot 2$ and $h(x):=x$ for all $x \neq 1,2$. Then $h(\alpha)=\pi_{N}(\alpha)=\alpha$ and, thus, according to (ii), (\{1\}, $\left.\{2,3,4,5\}\right)$ is an ambiguity partition with respect to $\alpha_{5}$. Furthermore, $h: \mathbb{N}^{*} \rightarrow \mathbb{N}^{*}$, defined by $h(1):=2, h(2):=\varepsilon$ and $h(x):=x$ for all $x \neq 1,2$, leads to the ambiguity partition $(\{1,2\},\{3,4,5\})$. In a similar manner, we can show that $(\{1,2,3\},\{4,5\})$ and $(\{1,2,3,4\},\{5\})$ are ambiguity partitions with respect to $\alpha_{5}$. In the latter ambiguity partition $(E, N)=(\{1,2,3,4\},\{5\})$, the set $E$ is of maximal size. We name those ambiguity partitions in the following technical definition that is useful in some of the subsequent proofs.

Definition 3.2. Let $\alpha \in \mathbb{N}^{+}$. An ambiguity partition $(E, N)$ with respect to $\alpha$ is called maximal if and only if every (other) ambiguity partition $\left(E^{\prime}, N^{\prime}\right)$ with respect to $\alpha$ satisfies $\left|E^{\prime}\right| \leq|E|$ and $\left|N^{\prime}\right| \geq|N|$.

Some simple observations on ambiguity partitions are made in the following proposition.

Proposition 3.3. Let $\alpha \in \mathbb{N}^{+}$.

(1) An ambiguity partition $(E, N)$ with respect to $\alpha$ is a partition of $\operatorname{var}(\alpha)$.

(2) If $\left(E^{\prime}, N^{\prime}\right)$ is an ambiguity partition with respect to $\alpha$ derived from an ambiguity partition $(E, N)$ by the rules of condition (ii) of Definition 3.1, then $\left|E^{\prime}\right|>|E|$ (and, thus, $\left|N^{\prime}\right|<|N|$ ).

(3) $\alpha$ is prolix if and only if there exists an ambiguity partition $(E, N)$ with respect to $\alpha$ such that $E \neq \emptyset$.

Proof. (1): Directly from Definition 3.1.

(2): Let $\alpha \in \mathbb{N}^{+},(E, N)$ be an ambiguity partition with respect to $\alpha$ and $h$ : $\mathbb{N}^{*} \rightarrow \mathbb{N}^{*}$ be a morphism that is nontrivial for $N$ and satisfies $h(\alpha)=\pi_{N}(\alpha)$. We show that $\{x \in N \mid h(x)=\varepsilon\}$ is nonempty, which proves the statement. Assume to the contrary that, for every $x \in N, h(x) \neq \varepsilon$. Hence, $(\star)\left|h\left(\pi_{N}(\alpha)\right)\right| \geq\left|\pi_{N}(\alpha)\right|$. Furthermore, since $h(\alpha)=\pi_{N}(\alpha)$, it follows that $|h(\alpha)|=\left|\pi_{N}(\alpha)\right|$ and, thus, $\left|h\left(\pi_{N}(\alpha)\right)\right| \leq\left|\pi_{N}(\alpha)\right|$. Consequently, with $(\star)$ we can conclude that $\left|h\left(\pi_{N}(\alpha)\right)\right|=$ $\left|\pi_{N}(\alpha)\right|$ and, hence, $h(e)=\varepsilon$ for every $e \in E$. Thus, $h\left(\pi_{N}(\alpha)\right)=\pi_{N}(\alpha)$. But then either $h$ is nonerasing and, thus, trivial for $N$ or there exists an $x \in N$ with $h(x)=\varepsilon$; both of these lead to a contradiction.

(3): We first show the only if direction. Hence, let $\alpha$ be prolix. Furthermore, let $E=\emptyset$ and $N=\operatorname{var}(\alpha)$. Then according to condition (i) of Definition 3.1, $(E, N)$ is an ambiguity partition with respect to $\alpha$. Since $\alpha$ is prolix, there exists a nontrivial morphism $h: \mathbb{N}^{*} \rightarrow \mathbb{N}^{*}$ satisfying $h(\alpha)=\alpha=\pi_{N}(\alpha)$. Consequently, $\left(E^{\prime}, N^{\prime}\right)$, $E^{\prime}, N^{\prime}$ defined as in condition (ii) of Definition 3.1, is an ambiguity partition of $\alpha$, and, according to statement (2) of the proposition, $E^{\prime} \neq \emptyset$. 
We proceed with the if direction. Let $(E, N)$ be an ambiguity partition with respect to $\alpha$ and let $E \neq \emptyset$. Since $E \neq \emptyset$, the rule of condition (ii) of Definition 3.1 must be applied at least once to derive $(E, N)$. Consequently, there must be an ambiguity partition $\left(E^{\prime}, N^{\prime}\right)$ which is derived from the partition $(\emptyset, \operatorname{var}(\alpha))$ by applying the rule of condition (ii). But this means that there exists a nontrivial morphism $h: \mathbb{N}^{*} \rightarrow \mathbb{N}^{*}$ satisfying $h(\alpha)=\pi_{\operatorname{var}(\alpha)}(\alpha)=\alpha$. Hence, we can conclude from Theorem 2.2 that $\alpha$ is prolix.

Finally, we state our main result on ambiguity partitions, which substantiates the usefulness of Definition 3.1 regarding the (un)ambiguity of morphisms.

Theorem 3.4. Let $\Sigma$ be an alphabet. Let $\alpha \in \mathbb{N}^{+}$and let $(E, N)$ be an ambiguity partition with respect to $\alpha$. Then every morphism $\sigma: \mathbb{N}^{*} \rightarrow \Sigma^{*}$ satisfying $\sigma(x) \neq \varepsilon$ for an $x \in E$ is ambiguous with respect to $\alpha$.

Proof. For $(E, N)=(\emptyset, \operatorname{var}(\alpha))$, there is no such morphism since $E=\emptyset$. Thus, the statement is true.

Now, let $\left(E^{\prime}, N^{\prime}\right)$ be an ambiguity partition derived from condition (ii) of Definition 3.1. Then there exists an ambiguity partition $(E, N)$ and a nontrivial morphism $h: \mathbb{N}^{*} \rightarrow \mathbb{N}^{*}$ satisfying $(\star) h(\alpha)=\pi_{N}(\alpha)$. Furthermore, $E^{\prime}=E \cup\{x \in N \mid$ $h(x)=\varepsilon\}$ and $N^{\prime}=\{x \in N \mid h(x) \neq \varepsilon\}$. We consider a morphism $\sigma: \mathbb{N}^{*} \rightarrow \Sigma^{*}$ satisfying $\sigma(x) \neq \varepsilon$ for an $x \in E^{\prime}$. At first, we assume that $(\star \star) \sigma(x)=\varepsilon$ for all $x \in E$ and $\sigma(n) \neq \varepsilon$ for an $n \in N$ with $h(n)=\varepsilon$. Let $\tau: \mathbb{N}^{*} \rightarrow \Sigma^{*}$ be the morphism defined by $\tau(x):=\sigma(h(x))$ for all $x \in \operatorname{var}(\alpha)$. Due to $(\star)$ and $(\star \star)$, $\tau(\alpha)=\sigma(h(\alpha))=\sigma\left(\pi_{N}(\alpha)\right)=\sigma(\alpha)$, but $\tau(n)=\varepsilon \neq \sigma(n)$. Consequently, $\sigma$ is ambiguous with respect to $\alpha$. If $\sigma(x) \neq \varepsilon$ for an $x \in E$, it follows by induction that $\sigma$ is ambiguous.

Hence, in order to find an unambiguous morphism for a pattern $\alpha \in \mathbb{N}^{*}$, we can initially investigate if $\alpha$ is succinct ( $c f$. Def. 2.1). In this case, there exists an unambiguous morphism $\sigma$ with respect to $\alpha$ ( $c f$. Theorem 2.3 - the explicit definition of $\sigma$ is given by Freydenberger et al. [4]). If $\alpha$ is prolix, according to Proposition 3.3, point (3), there exists an ambiguity partition $(E, N)$ with respect to $\alpha$. Moreover, Theorem 3.4 states a necessary condition for unambiguous morphisms with respect to $\alpha$ : such a morphism must "erase" all variables in $E$. Consequently, we do not only know that $\sigma$ must be erasing - which is already implied by Theorem 2.3 - but we even receive concrete evidence which variables at least must be mapped onto $\varepsilon$.

Additionally, as an immediate consequence of Theorem 3.4, we can state the following sufficient condition for what patterns there exists no unambiguous morphism:

Corollary 3.5. Let $\Sigma$ be an alphabet. Let $\alpha \in \mathbb{N}^{+}$. If $(\operatorname{var}(\alpha), \emptyset)$ is an ambiguity partition with respect to $\alpha$, no morphism $\sigma: \mathbb{N}^{*} \rightarrow \Sigma^{*}$ is unambiguous with respect to $\alpha$.

Proof. Directly from Theorem 3.4. 
This corollary covers, e.g., our example pattern $\alpha_{4}$ from Section 1.

In the following, we study whether or not the condition of Corollary 3.5 is necessary. It turns out that this depends on the size of the target alphabet of the morphism under consideration.

\section{Morphisms With An infinite target Alphabet}

If we consider an infinite target alphabet, the condition of Corollary 3.5 becomes characteristic:

Theorem 4.1. Let $\Sigma_{\infty}$ be an infinite alphabet and let $\alpha \in \mathbb{N}^{+}$. There is an unambiguous morphism $\sigma: \mathbb{N}^{*} \rightarrow \Sigma_{\infty}^{*}$ with respect to $\alpha$ if and only if $(\operatorname{var}(\alpha), \emptyset)$ is not an ambiguity partition with respect to $\alpha$.

Proof. We show the only if direction by contraposition: if $(\operatorname{var}(\alpha), \emptyset)$ is an ambiguity partition with respect to $\alpha$, it follows from Corollary 3.5 that no morphism is unambiguous with respect to $\alpha$.

To show the if part, assume that $N \neq \emptyset$ for every ambiguity partition $(E, N)$ with respect to $\alpha$. Let $(E, N)$ be a maximal ambiguity partition with respect to $\alpha$. W.l.o.g. let $\Sigma_{\infty}:=\mathbb{N}$. Furthermore, let the morphism $\sigma: \mathbb{N}^{*} \rightarrow \Sigma_{\infty}^{*}$ be defined by $\sigma:=\pi_{N}$. We show by contradiction that $\sigma$ is unambiguous with respect to $\alpha$. Assume that there exists a morphism $\tau: \mathbb{N}^{*} \rightarrow \Sigma_{\infty}^{*}$ satisfying $\tau(\alpha)=\sigma(\alpha)$ and $\tau(x) \neq \sigma(x)$ for an $x \in \operatorname{var}(\alpha)$. Then, $\tau(\alpha)=\sigma(\alpha)=\pi_{N}(\alpha)$ and, additionally, $\tau$ is nontrivial for $N$. But, according to condition (ii) of Definition 3.1, with $h:=\tau$, $\left(E^{\prime}, N^{\prime}\right)$ as defined in this condition is an ambiguity partition, too, satisfying $\left|E^{\prime}\right|>|E|$ and $\left|N^{\prime}\right|<|N|$ (cf. Prop. 3.3, point 2). This contradicts $(E, N)$ being maximal (cf. Def. 3.2). Thus, such a morphism $\tau$ cannot exist, and, hence, $\sigma$ is unambiguous with respect to $\alpha$.

Theorem 4.1 allows us to draw some conclusions on the decidability of the question of whether or not there exists an unambiguous morphism $\sigma: \mathbb{N}^{*} \rightarrow \Sigma_{\infty}^{*}$ for an arbitrary pattern $\alpha \in \mathbb{N}^{+}$.

Corollary 4.2. Let $\Sigma_{\infty}$ be an infinite alphabet. Then $\mathrm{AMB}_{\Sigma_{\infty}}$ is decidable.

Proof. Given a pattern $\alpha \in \mathbb{N}^{+}$, we can enumerate all (finitely many) ambiguity partitions with respect to $\alpha$ since the existence of a morphism $h$ as requested by condition (ii) of Definition 3.1 can be checked effectively. Finally, $\alpha \in \mathrm{AMB}_{\Sigma_{\infty}}$ if and only if $(\operatorname{var}(\alpha), \emptyset)$ is an ambiguity partition with respect to $\alpha$ (cf. Thm. 4.1).

Unfortunately, however, this decision problem is NP-complete.

Theorem 4.3. Let $\Sigma_{\infty}$ be an infinite alphabet. The problem of deciding $\mathrm{AMB}_{\Sigma_{\infty}}$ is NP-complete. 
Proof. We first show that $\mathrm{AMB}_{\Sigma_{\infty}}$ is in NP. For this purpose, we describe a nondeterministic Turing machine $\mathrm{M}$ that accepts $\mathrm{AMB}_{\Sigma_{\infty}}$ : first, $\mathrm{M}$ writes nondeterministically a sequence $S:=\left(E_{1}, N_{1}, h_{1}\right),\left(E_{2}, N_{2}, h_{2}\right), \ldots,\left(E_{k}, N_{k}, h_{k}\right)$ for a nondeterministically chosen $k \in\{1,2, \ldots,|\operatorname{var}(\alpha)|+1\}$ and $E_{i}, N_{i} \subseteq \operatorname{var}(\alpha), 1 \leq i \leq k$. The $h_{i}$ correspond to morphisms $h_{i}: \mathbb{N}^{*} \rightarrow \mathbb{N}^{*}$ and can each be given as a list $\left[x_{1}, h\left(x_{1}\right), x_{2}, h\left(x_{2}\right), \ldots, x_{|\operatorname{var}(\alpha)|}, h\left(x_{|\operatorname{var}(\alpha)|}\right)\right]$. Clearly, the length of the sequence $S$ is polynomial in $|\alpha|$. Afterwards, we check if $S$ is "compatible" with the definition of the ambiguity partition, which means that $\left(E_{1}, N_{1}\right):=(\emptyset, \operatorname{var}(\alpha))$ as well as, for every $i, 2 \leq i \leq k,\left(E_{i}, N_{i}\right)$ is a partition of $\operatorname{var}(\alpha)$ and $(E, N):=\left(E_{i-1}, N_{i-1}\right)$, $h=h_{i}$ and $\left(E^{\prime}, N^{\prime}\right):=\left(E_{i}, N_{i}\right)$ satisfy condition (ii) of Definition 3.1. It can be verified with little effort that this check can be done in polynomial time. We let $\mathrm{M}$ go into the accepting state if and only if this check is successful and $\left(E_{k}, N_{k}\right)=(\operatorname{var}(\alpha), \emptyset)$. Due to Proposition 3.3 (ii), if the check is successful, $\left|E_{i}\right|>\left|E_{i+1}\right|$ holds for every $i \in\{1,2, \ldots, k-1\}$. Thus, if $(\operatorname{var}(\alpha), \emptyset)$ is an ambiguity partition with respect to $\alpha$, it can be "reached" by a sequence $S$ of maximal length $|\operatorname{var}(\alpha)|+1$. Consequently, M accepts exactly those patterns $\alpha$ (in polynomial time) such that $(\operatorname{var}(\alpha), \emptyset)$ is an ambiguity partition with respect to $\alpha$. Due to Theorem 4.1, these are exactly the patterns in $\mathrm{AMB}_{\Sigma_{\infty}}$.

In order to show that $\mathrm{AMB}_{\Sigma_{\infty}}$ is NP-hard, we reduce the following problem to $\mathrm{AMB}_{\Sigma_{\infty}}$.

Morphism Problem/Match Test: the problem of deciding $\operatorname{MATCH}_{\{a, b\}}:=\{(\alpha$, $w) \mid$ there exists a morphism $\sigma: \mathbb{N}^{*} \rightarrow\{\mathrm{a}, \mathrm{b}\}^{*}$ such that $\left.\sigma(\alpha)=w\right\}$ is NPcomplete ( $c f$. Ehrenfeucht and Rozenberg [2]).

We define a function $f$ for which we shall prove the following:

(a) $f$ is computable in polynomial time;

(b) $(\alpha, w) \in \operatorname{MATCH}_{\{\mathrm{a}, \mathrm{b}\}}$ if and only if $f(\alpha, w) \in \mathrm{AMB}_{\Sigma_{\infty}}$.

In order to define $f(\alpha, w)$, we need the following auxiliary definitions: let $\alpha \in \mathbb{N}^{*}$ and $w \in \Sigma^{*}$. W. l. o. g. let $\operatorname{var}(\alpha) \subseteq\{3,5,7, \ldots\}$. Furthermore, let $d: \mathbb{N}^{*} \rightarrow \mathbb{N}^{*}$ and $\sigma_{\text {inv }}: \Sigma^{*} \rightarrow \mathbb{N}^{*}$ be morphisms, defined as follows: let

$$
d(x):=x \cdot(x+1),
$$

where $\cdot$ refers to the concatenation, and

$$
\sigma_{\text {inv }}(c):= \begin{cases}1, & c=\mathrm{a} \\ 2, & c=\mathrm{b}\end{cases}
$$

If $\sigma_{\text {inv }}(w)$ is succinct, we set $\beta:=\sigma_{\text {inv }}(w)$, otherwise (if $\sigma_{\operatorname{inv}}(w)$ is prolix), according to Theorem 2.2, there exists a morphism $g: \mathbb{N}^{*} \rightarrow \mathbb{N}^{*}$ such that $g\left(\sigma_{\text {inv }}(w)\right)=$ $\sigma_{\text {inv }}(w)$ and $g(i) \neq i$ for an $i \in\{1,2\}$. It particularly follows that $g(x) \neq \varepsilon$ and $g(y)=\varepsilon$ for $\{x, y\}=\{1,2\}$. In this case, let $\beta:=\pi_{\{x\}}\left(\sigma_{\mathrm{inv}}(w)\right)$.

Finally, let $f(\alpha, w):=d(\alpha) \beta \beta d(\alpha)$.

Proof of (a). It is clear that $d(\alpha)$ and $\sigma_{\text {inv }}(\alpha)$ can be constructed in polynomial time. It remains to show that we can also efficiently check if $\sigma_{\text {inv }}(w)$ is prolix. 
It can be verified with a little effort that $\sigma_{\text {inv }}(w)$ is prolix if and only if, for $\{x, y\}=\{1,2\}, \sigma_{\mathrm{inv}}(w)=\left(y^{l} x y^{r}\right)^{s}, l+r \geq 1, s \geq 1$. This can be checked in polynomial time (by scanning $\sigma_{\text {inv }}(w)$ from left to right, once assuming $x=1$, $y=2$, once vice versa).

Proof of (b). We first show the only if part. Let $(\alpha, w) \in \operatorname{MATCH}_{\{\mathbf{a}, \mathbf{b}\}}$. Hence, there exists a morphism $h^{\prime}: \mathbb{N}^{*} \rightarrow \Sigma^{*}$ such that $h^{\prime}(\alpha)=w$. Without loss of generality, let $h^{\prime}(x):=\varepsilon$ for every $x \in\{1,2\} \cup\{4,6,8, \ldots\}$. Thus, $h^{\prime}(\beta)=\varepsilon$ and $h^{\prime}(d(\alpha))=h^{\prime}(\alpha)=w$. Because of the structure of $f(\alpha, w)$ and the fact that $d(\alpha)$ is a pattern with the ambiguity partition $(\operatorname{var}(d(\alpha)), \emptyset)$, we can verify that $(E, N):=(\operatorname{var}(d(\alpha)), \operatorname{var}(\beta))$ is an ambiguity partition with respect to $f(\alpha, w)$. We consider two disjoint cases.

Case 1: $\sigma_{\mathrm{inv}}(w)$ is succinct. Then $(E, N)$ and $h:=\sigma_{\mathrm{inv}} \circ h^{\prime}$ satisfy condition (ii) of Definition 3.1 since

$$
\begin{aligned}
h(f(\alpha, w)) & =h(d(\alpha)) \cdot h(\beta \beta) \cdot h(d(\alpha)) \\
& =\sigma_{\mathrm{inv}}\left(h^{\prime}(d(\alpha))\right) \cdot \varepsilon \cdot \sigma_{\mathrm{inv}}\left(h^{\prime}(d(\alpha))\right) \\
& =\sigma_{\mathrm{inv}}(w) \cdot \sigma_{\mathrm{inv}}(w)=\beta \cdot \beta=\pi_{N}(f(\alpha, w)) .
\end{aligned}
$$

Moreover, $h$ is nontrivial for $N$.

Case 2: $\sigma_{\mathrm{inv}}(w)$ is prolix. Then $(E, N)$ and $h:=\pi_{\{x\}} \circ \sigma_{\mathrm{inv}} \circ h^{\prime}$ satisfy condition (ii) of Definition 3.1 since

$$
\begin{aligned}
h(f(\alpha, w)) & =h(d(\alpha)) \cdot h(\beta \beta) \cdot h(d(\alpha)) \\
& =\pi_{\{x\}}\left(\sigma_{\text {inv }}\left(h^{\prime}(d(\alpha))\right)\right) \cdot \varepsilon \cdot \pi_{\{x\}}\left(\sigma_{\text {inv }}\left(h^{\prime}(d(\alpha))\right)\right) \\
& =\pi_{\{x\}}\left(\sigma_{\text {inv }}(w)\right) \cdot \pi_{\{x\}}\left(\sigma_{\text {inv }}(w)\right)=\beta \cdot \beta=\pi_{N}(f(\alpha, w)) .
\end{aligned}
$$

Moreover, $h$ is nontrivial for $N$.

In both cases, $h(x)=\varepsilon$ for every $x \in \operatorname{var}(\beta)$. Thus $(\operatorname{var}(f(\alpha, w)), \emptyset)$ is an ambiguity partition with respect to $f(\alpha, w)$. Hence, according to Theorem 4.1, there is no unambiguous morphism with respect to $f(\alpha, w)$. This proves the only if part.

We now show the if part by contraposition. Let $(\alpha, w) \notin \operatorname{MATCH}_{\{\mathbf{a}, \mathbf{b}\}}$. Without loss of generality, let $\{\mathrm{a}, \mathrm{b}\} \subseteq \Sigma_{\infty}$. We shall prove that the morphism $\sigma: \mathbb{N}^{*} \rightarrow$ $\Sigma_{\infty}^{*}$, defined by $\sigma(1):=\mathrm{a}, \sigma(2):=\mathrm{b}, \sigma(x):=\varepsilon, x \in \mathbb{N} \backslash\{1,2\}$, is unambiguous with respect to $f(\alpha, w)$. Assume to the contrary that there exists a morphism $\tau: \mathbb{N}^{*} \rightarrow$ $\Sigma_{\infty}^{*}$ satisfying $\sigma(f(\alpha, w))=\tau(f(\alpha, w))$ and $\sigma(i) \neq \tau(i)$ for an $i \in \operatorname{var}(f(\alpha, w))$. We consider the following cases:

Case 1: $\operatorname{var}(\beta)=\{x\}$. Hence, either $\left|\operatorname{var}\left(\sigma_{\text {inv }}(w)\right)\right|=1$ or $\sigma_{\text {inv }}(w)$ is prolix. In the former case, let $g$ be the identity morphism, in the latter case, let $g$ be the morphism as defined above (below the definition of $\sigma_{\text {inv }}$ ).

Case 1.1: $\tau(x) \neq \varepsilon$. Thus, either $\tau(f(\alpha, w)) \neq \sigma(f(\alpha, w))$ or $\tau(f(\alpha, w))=$ $\sigma(f(\alpha, w)), \tau(x)=\sigma(x)$ and, hence, $\tau(i)=\sigma(i)$ for every $i \in \operatorname{var}(f(\alpha, w))$, which contradicts the assumption. 
Case 1.2: $\tau(x)=\varepsilon$. Then $\tau(d(\alpha))=\sigma(\beta)$ and, hence, $\sigma \circ g \circ \sigma_{\text {inv }} \circ \tau \circ d(\alpha)=$ $\sigma \circ g \circ \sigma_{\text {inv }} \circ \sigma(\beta)=w$ since $\sigma_{\text {inv }}(\sigma(\beta))=\beta, g(\beta)=\sigma_{\text {inv }}(w)$ and $\sigma\left(\sigma_{\text {inv }}(w)\right)=w$. Thus, $(\alpha, w) \in \operatorname{MATCH}_{\{\mathbf{a}, \mathbf{b}\}}$, which is a contradiction.

Case 2: $\operatorname{var}(\beta)=\{x, y\}$. Hence, $\beta=\sigma_{\text {inv }}(w)$ is succinct.

Case 2.1: $\tau(i)=\varepsilon$ for all $i \in \operatorname{var}(d(\alpha))$. Then, $\sigma_{\text {inv }}(\tau(\beta))=\beta$ and $\sigma_{\text {inv }} \circ \tau$ is nontrivial for $\operatorname{var}(\beta)$ since $\tau(i) \neq \sigma(i)$ for an $i \in \operatorname{var}(f(\alpha, w))$ is required. Thus, $\beta$ is prolix, which contradicts $\beta$ being succinct.

Case 2.2: $\tau(i) \neq \varepsilon$ for an $i \in \operatorname{var}(d(\alpha))$. Let $\tau(i)=\ldots$ a $\ldots$ (the case $\tau(i)=$ $\ldots \mathrm{b} \ldots$ is analogous $)$. Then, $\tau(1)=\varepsilon$ since otherwise $\mid \tau\left(\left.f(\alpha, w)\right|_{\mathrm{a}}>\mid \sigma\left(\left.f(\alpha, w)\right|_{\mathrm{a}}\right.\right.$. Assume that $\tau(2) \neq \varepsilon$. With $|\tau(f(\alpha, w))|_{\mathrm{a}}=|\sigma(f(\alpha, w))|_{\mathrm{a}}$ and $|\tau(f(\alpha, w))|_{\mathrm{b}}=$ $|\sigma(f(\alpha, w))|_{\mathrm{b}}$, it follows that $\tau(2)=\mathrm{b}$. Due to the structure of $f(\alpha, w)$, we have $\tau(f(\alpha, w))=\ldots \sigma\left(\pi_{\{2\}}(\beta)\right)^{2} \ldots$, but $\sigma\left(\pi_{2}(\beta)\right)^{2}$ is not a factor of $\sigma(f(\alpha, w))$. This contradicts the existence of a morphism $\tau$ with $\tau\left(j_{1}\right) \neq \varepsilon$ and $\tau\left(j_{2}\right)=\varepsilon,\left\{j_{1}, j_{2}\right\}=$ $\{1,2\}$. Consequently, $\tau(1)=\tau(2)=\varepsilon$. Thus, $\tau(d(\alpha))=\sigma(\beta)=w$ and, hence, $(\alpha, w) \in \operatorname{MATCH}_{\{\mathrm{a}, \mathrm{b}\}}$, which is again a contradiction.

Consequently, such a morphism $\tau$ cannot exist. Hence, $\sigma$ is unambiguous with respect to $\alpha$, and this implies $(\alpha, w) \notin \mathrm{AMB}_{\Sigma_{\infty}}$.

The proof of Theorem 4.3 allows us to receive a result on the minimal complexity of the $\mathrm{AMB}_{\Sigma}$ decision problem for a finite alphabet $\Sigma$, although it is still open if in this case $\mathrm{AMB}_{\Sigma}$ is decidable at all.

Corollary 4.4. Let $\Sigma$ be an finite alphabet, $|\Sigma| \geq 2$. The problem of deciding $\mathrm{AMB}_{\Sigma}$ is NP-hard.

Proof. We can use the same reduction function $f$ as in the proof of Theorem 4.3 as it is sufficient to have $\{a, b\} \subseteq \Sigma$, which we can assume without loss of generality. bet.

In the next section, we continue to study morphisms with a finite target alpha-

\section{Morphisms With a finite target Alphabet}

Although the ambiguity of morphisms with an infinite target alphabet regarding prolix patterns and, thus, erasing morphisms, offers a wider spectrum of results than regarding succinct patterns for which the identity morphism is unambiguous (as a consequence of Thm. 2.2), the much more interesting question reads as follows: for which patterns $\alpha$ do there exist unambiguous morphisms mapping to words over a finite target alphabet $\Sigma$, especially if $|\Sigma|<|\operatorname{var}(\alpha)|$, e.g. $|\Sigma|=$ 2? Unfortunately, as to be shown below, Theorem 4.1 does not hold for finite alphabets. In order to find an explanation for this situation, we take a closer look at those other morphisms $\tau$ which exist if $\sigma$ is ambiguous ( $c f$. example pattern $\alpha_{5}$ from the beginning of Sect. 3): if there is an ambiguity partition $(E, N)$ with respect to $\alpha$ and $\sigma(e) \neq \varepsilon$ for an $e \in E$, another morphism $\tau$ with $\tau(\alpha)=\sigma(\alpha)$ can generate the whole image of $e$ under $\sigma$ with another variable 
$e^{\prime} \neq e$, i.e. $\tau\left(e^{\prime}\right)=\ldots \sigma(e) \ldots$ and $\tau(e)=\varepsilon$. However, this is not the only "type" of ambiguity that can occur: referring to example pattern $\alpha_{1}$ from the introduction, we observe that in this case $\tau$ only generates partial images of variables by $\sigma$. This phenomenon must be taken into account whenever we look for an unambiguous morphism $\sigma: \mathbb{N}^{*} \rightarrow \Sigma^{*}$ with respect to a pattern $\alpha$, provided that $\Sigma$ is finite and $|\Sigma|<|\operatorname{var}(\alpha)|$, since not every variable can be mapped to a different letter in $\Sigma$.

The following theorem states some fundamental insights into the ambiguity of morphisms with a finite target alphabet and, in particular, shows that Theorem 4.1 does not hold for finite alphabets.

Theorem 5.1. Let $k \in \mathbb{N}$ and $\Sigma_{k}, \Sigma_{k+1}$ be finite alphabets with $k$ and $k+1$ letters, respectively. There exists a pattern $\alpha \in \mathbb{N}^{+}$such that

(i) $(\operatorname{var}(\alpha), \emptyset)$ is not an ambiguity partition with respect to $\alpha$;

(ii) no morphism $\sigma: \mathbb{N}^{*} \rightarrow \Sigma_{k}^{*}$ is unambiguous with respect to $\alpha$; and

(iii) there exists an unambiguous morphism $\sigma^{\prime}: \mathbb{N}^{*} \rightarrow \Sigma_{k+1}^{*}$ with respect to $\alpha$.

Proof. We give a pattern $\alpha_{k} \in \mathbb{N}^{+}$that satisfies the conditions (i)-(iii). For every $i, i^{\prime}, j, j^{\prime} \in\{1,2, \ldots, k+1\}, i \neq j, i^{\prime} \neq j^{\prime}$, let $x_{\{i, j\}} \in \mathbb{N} \backslash\{1,2, \ldots, k+1\}$ and $x_{\{i, j\}} \neq x_{\left\{i^{\prime}, j^{\prime}\right\}}$ if and only if $\{i, j\} \neq\left\{i^{\prime}, j^{\prime}\right\}$. We define $\alpha_{k}$ as follows:

$$
\begin{aligned}
\alpha_{k} & :=\beta_{1} \beta_{2}[\ldots] \beta_{k+1} \beta_{k+1} \beta_{k}[\ldots] \beta_{1} \text {, with } \\
\beta_{i} & :=i \cdot \prod_{\substack{1 \leq j \leq k+1, j \neq i}} x_{\{i, j\}} .
\end{aligned}
$$

For instance,

$$
\begin{aligned}
\alpha_{2}= & 1 \cdot x_{\{1,2\}} \cdot x_{\{1,3\}} \cdot 2 \cdot x_{\{2,1\}} \cdot x_{\{2,3\}} \cdot 3 \cdot x_{\{3,1\}} \cdot x_{\{3,2\}} \\
& 3 \cdot x_{\{3,1\}} \cdot x_{\{3,2\}} \cdot 2 \cdot x_{\{2,1\}} \cdot x_{\{2,3\}} \cdot 1 \cdot x_{\{1,2\}} \cdot x_{\{1,3\}}
\end{aligned} .
$$

Note that, e.g., $x_{\{1,2\}}=x_{\{2,1\}}$ since $\{1,2\}=\{2,1\}$. Hence, $\left|\operatorname{var}\left(\alpha_{2}\right)\right|=6$. Thus, $\alpha_{2}$ could equal $1 \cdot 4 \cdot 5 \cdot 2 \cdot 4 \cdot 6 \cdot 3 \cdot 5 \cdot 6 \cdot 3 \cdot 5 \cdot 6 \cdot 2 \cdot 4 \cdot 6 \cdot 1 \cdot 4 \cdot 5$. This pattern may be consulted for a better understanding of the proof although the subsequent argumentation deals with the general pattern $\alpha_{k}$.

Proof of (i): Let $I:=\{1,2, \ldots, k+1\}$. We show that, for every ambiguity partition $(E, N)$ with respect to $\alpha_{k}$ and every $i \in I, i \notin E$. Assume to the contrary that there exists an ambiguity partition $\left(E^{\prime}, A^{\prime}\right)$ with respect to $\alpha_{k}$ such that $i \in E^{\prime}$ for an $i \in I$. Then, there exist ambiguity partitions $\left(E_{1}, N_{1}\right)$ and $\left(E_{2}, N_{2}\right)$ such that

(1) for every $i \in I, i \notin E_{1}$;

(2) there exists a $j \in I$ with $j \in E_{2}$; and

(3) there exists a morphism $h: \mathbb{N}^{*} \rightarrow \mathbb{N}^{*}$ that is nontrivial for $N_{1}$ and satisfies $h\left(\alpha_{k}\right)=\pi_{N_{1}}\left(\alpha_{k}\right)$ and $h(j)=\varepsilon$, according to condition (ii) of Definition 3.1. Thus, following the inductive conception of Definition 3.1, the "step" from $\left(E_{1}, N_{1}\right)$ to $\left(E_{2}, N_{2}\right)$ is the first one where an $i \in I$ is included into the $E$-set of an ambiguity partition. Let $h^{\prime}: \mathbb{N}^{*} \rightarrow \mathbb{N}^{*}$ be defined by $h^{\prime}:=\pi_{I} \circ h$. We can verify 
$h^{\prime}\left(\pi_{I}\left(\alpha_{k}\right)\right)=\pi_{I}\left(\alpha_{k}\right)$ as follows: the above condition (1) implies $I \subseteq N_{1}$. Furthermore, since every $x_{\{l, m\}}, 1 \leq l, m \leq k+1, l \neq m$ occurs four times in $\alpha_{k}$ while every $l \in I$ occurs only twice, every occurrence of an $l \in I$ in $h\left(\alpha_{k}\right)$ must be generated by $h$ applied to an $m \in I$. Additionally, $h^{\prime}(j)=\varepsilon$ and, thus, $\pi_{I}\left(\alpha_{k}\right)$ is a fixed point of the nontrivial morphism $h^{\prime}$. Consequently, $\pi_{I}\left(\alpha_{k}\right)$ is prolix ( $c f$. Thm. 2.2), which is a contradiction since there exists no factorisation of $\pi_{I}\left(\alpha_{k}\right)$ as required by Definition 2.1. Thus, for every ambiguity partition $(E, N)$ with respect to $\alpha_{k}$ and every $i \in I, i \notin E$ and, hence, $\left(\operatorname{var}\left(\alpha_{k}\right), \emptyset\right)$ is not an ambiguity partition with respect to $\alpha_{k}$.

Proof of (ii): Assume to the contrary that there exists an unambiguous morphism $\sigma: \mathbb{N}^{*} \rightarrow \Sigma_{k}^{*}$ with respect to $\alpha$. Let $N:=\{1,2, \ldots, k+1\}, E:=\operatorname{var}\left(\alpha_{k}\right) \backslash N$. Since the morphism $h: \mathbb{N}^{*} \rightarrow \mathbb{N}^{*}$ defined by

$$
h(i):= \begin{cases}\beta_{i}, & \text { if } 1 \leq i \leq k+1 \\ \varepsilon, & \text { else }\end{cases}
$$

is nontrivial for $N$ and satisfies $h\left(\alpha_{k}\right)=\alpha_{k},(E, N)$ is an ambiguity partition with respect to $\alpha$ (according to Def. 3.1). Thus, it follows from Theorem 3.4 that $\sigma(e)=\varepsilon$ for every $e \in E$. Hence, exactly one of the following cases must occur.

Case 1. $\sigma(n)=\varepsilon$ for an $n \in N$. If $n=1$ then the morphism $\tau: \mathbb{N}^{*} \rightarrow \Sigma_{k}^{*}$, defined by $\tau(1):=\sigma(2), \tau(2):=\varepsilon, \tau(x):=\sigma(x)$ for all $x \in N \backslash\{1,2\}, \tau(y)=\varepsilon$ for all $y \in E$, satisfies $\tau\left(\alpha_{k}\right)=\sigma\left(\alpha_{k}\right)$ and, thus, contradicts $\sigma$ being unambiguous with respect to $\alpha_{k}$. If $n>1$ then the morphism $\tau: \mathbb{N}^{*} \rightarrow \Sigma_{k}^{*}$, defined by $\tau(n)=\sigma(n-1)$, $\tau(n-1)=\varepsilon, \tau(x)=\sigma(x)$ for all $x \in N \backslash\{n-1, n\}, \tau(y)=\varepsilon$ for all $y \in E$, satisfies $\tau\left(\alpha_{k}\right)=\sigma\left(\alpha_{k}\right)$ and, thus, contradicts $\sigma$ being unambiguous with respect to $\alpha_{k}$.

Case 2. $\sigma(n) \neq \varepsilon$ for every $n \in N$. Since $|N|=k+1>\left|\Sigma_{k}\right|$, the image of two variables under $\sigma$ must end with the same letter, i.e. there exist $i, j \in N$, $w_{i}, w_{j} \in \Sigma_{k}^{*}$ and a letter $c \in \Sigma_{k}$ such that $\sigma(i)=w_{i} c$ and $\sigma(j)=w_{j} c$. We define a morphism $\tau: \mathbb{N}^{*} \rightarrow \Sigma_{k}^{*}$ as $\tau(i):=w_{i}, \tau(j):=w_{j}, \tau\left(x_{\{i, j\}}\right):=c, \tau(x):=\sigma(x)$ for all $x \in N \backslash\{i, j\}, \tau(y):=\varepsilon$ for all $y \in E \backslash\left\{x_{\{i, j\}}\right\}$. This morphism satisfies $\tau\left(\alpha_{k}\right)=\sigma\left(\alpha_{k}\right)$ and, thus, contradicts $\sigma$ being unambiguous with respect to $\alpha_{k}$.

Consequently, there exists no unambiguous morphism $\sigma: \mathbb{N}^{*} \rightarrow \Sigma_{k}^{*}$ with respect to $\alpha_{k}$.

Proof of (iii). Let $\Sigma_{k+1}=N:=\{1,2, \ldots, k+1\}$ and $E:=\operatorname{var}\left(\alpha_{k}\right) \backslash N$. We show that the morphism $\sigma^{\prime}: \mathbb{N}^{*} \rightarrow \Sigma_{k+1}^{*}$, defined by $\sigma^{\prime}(n):=n$ for every $n \in N$, $\sigma^{\prime}(e):=\varepsilon$ for every $e \in E$, is unambiguous with respect to $\alpha_{k}$. Assume to the contrary that there exists a morphism $\tau: \mathbb{N}^{*} \rightarrow \Sigma_{k+1}^{*}$ satisfying $\tau\left(\alpha_{k}\right)=\sigma^{\prime}\left(\alpha_{k}\right)$ and $\tau(i) \neq \sigma^{\prime}(i)$ for an $i \in \operatorname{var}\left(\alpha_{k}\right)$. Since $\left|\sigma^{\prime}\left(\alpha_{k}\right)\right|_{n}=2$ for every $n \in N$ but $\left|\alpha_{k}\right|_{e}=4$ for every $e \in E, \tau(e)=\varepsilon$ for every $e \in E$. Hence, $\tau\left(\pi_{N}\left(\alpha_{k}\right)\right)=\tau\left(\alpha_{k}\right)=$ $\sigma^{\prime}\left(\alpha_{k}\right)=\pi_{N}\left(\alpha_{k}\right)$ and, thus, $\pi_{N}\left(\alpha_{k}\right)$ is a fixed point of $\tau$. Due to $\tau(i) \neq \sigma(i)=i$, $\tau$ is nontrivial. Consequently, $\pi_{N}\left(\alpha_{k}\right)$ is prolix (cf. Thm. 2.2). But there exists no factorisation of $\pi_{N}\left(\alpha_{k}\right)$ as required by Definition 2.1. Hence, $\pi_{N}\left(\alpha_{k}\right)$ is not prolix, which contradicts the existence of $\tau$. Thus, $\sigma^{\prime}$ is unambiguous with respect to $\alpha_{k}$. 
Theorem 5.1 allows us to compare the sets of patterns for which there exist no unambiguous morphisms when different target alphabets are chosen.

Corollary 5.2. Let $\Sigma, \Sigma^{\prime}$ be finite alphabets, $|\Sigma|<\left|\Sigma^{\prime}\right|$. Then $\mathrm{AMB}_{\Sigma} \supset \mathrm{AMB}_{\Sigma^{\prime}}$.

Proof. Referring to the definition of $\mathrm{AMB}_{\Sigma}$, we can verify $\mathrm{AMB}_{\Sigma} \supseteq \mathrm{AMB}_{\Sigma^{\prime}}$. $\mathrm{AMB}_{\Sigma} \neq \mathrm{AMB}_{\Sigma^{\prime}}$ follows from Theorem 5.1 (iii).

This establishes a novel and rather unexpected aspect in the research on the ambiguity of morphisms. While, for a pattern $\alpha$, there exists an unambiguous nonerasing morphism if and only if $\alpha$ is succinct - no matter which $\Sigma$ with $|\Sigma| \geq 2$ is chosen ( $c f$. Thm. 2.3), concerning erasing morphisms, this question strongly depends on the size of $\Sigma$. This also means that the techniques introduced in Freydenberger et al. [4] are of limited use when exploring the ambiguity of erasing morphisms.

In addition to this, the approach studied by previous literature of first defining $\Sigma$ and then examining $\mathrm{AMB}_{\Sigma}$ could also be reversed as follows: given an arbitrary pattern $\alpha$, which is the minimal size of $\Sigma$ such that there exists an unambiguous morphism $\sigma: \mathbb{N}^{*} \rightarrow \Sigma^{*}$ if such a morphism exists at all?

We conclude this section with a result that adds some restrictions to the class of patterns considered in order to make the criterion of Theorem 4.1 characteristic for any finite alphabet with at least two letters.

Theorem 5.3. Let $\Sigma$ be an alphabet, $|\Sigma| \geq 2$. Let $\alpha \in \mathbb{N}^{+}$, let $(E, N)$ be a maximal ambiguity partition with respect to $\alpha$ and let, for every $i \in N, \alpha=\ldots i i$...

There exists an unambiguous morphism $\sigma: \mathbb{N}^{*} \rightarrow \Sigma^{*}$ with respect to $\alpha$ if and only if $N \neq \emptyset$.

Proof. Let $n:=|\alpha|$.

We first prove the only if part by contraposition: if $N=\emptyset$ then $(\operatorname{var}(\alpha), \emptyset)$ is an ambiguity partition with respect to $\alpha$ and, thus, according to Corollary 3.5, there exists no unambiguous morphism with respect to $\alpha$.

To show the if part, let $N \neq \emptyset$. Furthermore, let $\sigma: \mathbb{N}^{*} \rightarrow\{\mathrm{a}, \mathrm{b}\}^{*}$ be a morphism defined by

$$
\sigma(i):= \begin{cases}\mathrm{ab}^{n i+1} \mathrm{aab}^{n i+2} \mathrm{a} \ldots \mathrm{ab}^{n(i+1)} \mathrm{a}, & \text { if } i \in N \\ \varepsilon, & \text { else. }\end{cases}
$$

Note that, for variables in $N, \sigma$ corresponds to the morphism $\tau_{k, a, b}$ as introduced by Jiang et al. [7].

We now prove that $\sigma$ is unambiguous with respect to $\alpha$ : assume to the contrary that there exists a morphism $\tau: \mathbb{N}^{*} \rightarrow\{\mathrm{a}, \mathrm{b}\}^{*}$ such that $(\star) \tau(\alpha)=\sigma(\alpha)$ and $\tau(j) \neq \sigma(j)$ for a $j \in \operatorname{var}(\alpha)$.

Case 1. For all $i \in N$, there exists an $x_{i} \in\{n i+1, n i+2, \ldots, n(i+1)\}$ such that $\tau(i)=\ldots \mathrm{ab}^{x_{i}} \mathrm{a} \ldots$. Assume that $\tau(i)=\sigma(i)$ for all $i \in N$. Then either $\tau(\alpha) \neq \sigma(\alpha)$ or $\tau(j)=\sigma(j)$ for all $j \in \operatorname{var}(\alpha)$, which both contradicts $(\star)$. Thus, $\tau(i) \neq \sigma(i)$ for an $i \in N$. Since $\alpha=\ldots i i \ldots, \tau(i i)$ is a factor of $\tau(\alpha)$. But - due to $\tau(i) \neq \sigma(i)-$ it can be verified that $\tau(i i)$ is not a factor of $\sigma(\alpha)$, which contradicts $\tau(\alpha)=\sigma(\alpha)$. 
Case 2. There exists a $j \in N$ such that, for all $x_{j} \in\{n j+1, n j+2, \ldots, n(j+1)\}$, $\tau(j) \neq \ldots \mathrm{ab}^{x_{j}} \mathrm{a} \ldots$. The following reasoning is directly taken from Jiang et al. [7]: because, for every $i \in N, \sigma(i)$ contains $n=|\alpha|$ "segments" of the form $\mathrm{ab}^{m} \mathrm{a}$, $m \in \mathbb{N}$, it follows that, for all $i \in N$, there exist $x_{i} \in\{n i+1, n i+2, \ldots, n(i+1)\}$ and $y \in \operatorname{var}(\alpha)$ such that $\tau(y)=\ldots \mathrm{ab}^{x_{i}} \mathrm{a} \ldots$ For every $i \in N$, we choose such an $x_{i}$ and define a morphism $h: \mathbb{N}^{*} \rightarrow \mathbb{N}^{*}$ for every $y \in \operatorname{var}(\alpha)$ as follows:

$$
h(y):= \begin{cases}i_{1} i_{2} \ldots i_{k}, & \text { if } \tau(y)=w_{0} \mathrm{ab}^{x_{i_{1}}} \mathrm{a} w_{1} \mathrm{ab}^{x_{i_{2}}} \mathrm{a} w_{2} \ldots \mathrm{ab}^{x_{i_{k}}} w_{k}, k \in \mathbb{N} \\ & \text { satisfying } w_{i} \in \Sigma^{*} \text { and } w_{i} \neq \ldots \mathrm{ab}^{x_{j}} \mathrm{a} \ldots \\ & \text { for all } i \in\{0,1, \ldots, k\} \text { and all } j \in N \\ & \text { else. }\end{cases}
$$

$h$ is nontrivial for $N$ because $h$ is nontrivial for $\{j\}$. Furthermore, $h(\alpha)=\pi_{N}(\alpha)$ since, for every $i \in N$, there exists exactly one corresponding $x_{i}$. But, according to condition (ii) of Definition 3.1, $\left(E^{\prime}, N^{\prime}\right)$ as defined in this condition, is an ambiguity partition, too, satisfying $\left|E^{\prime}\right|>|E|$ and $\left|N^{\prime}\right|<|N|$ (cf. Prop. 3.3, point 2). This contradicts the assumption that $(E, N)$ is maximal (cf. Def. 3.2).

Consequently, such a morphism $\tau$ cannot exist since exactly one of the two cases must occur. Thus, $\sigma$ is unambiguous with respect to $\alpha$.

Note that, with the proof technique of Theorem 5.3, conditions similar to $\alpha=$ $\ldots i i \ldots$ for every $i \in N$ can certainly be found since it is sufficient for any such condition to let case 1 in the proof lead to a contradiction.

\section{Conclusions}

In the present paper, we have initiated the systematic research on the ambiguity of erasing morphisms. We have shown that a certain structure of a pattern, namely the existence of an ambiguity partition with respect to this pattern, strongly contributes to the ambiguity of morphisms applied to the pattern. In the case of infinite target alphabets, this structure even characterises the ambiguity of erasing morphisms and allows conclusions on the complexity of algorithms that decide $\mathrm{AMB}_{\Sigma_{\infty}}$ to be drawn. Concerning finite target alphabets, the rather intricate situation has been identified that the ambiguity of morphisms is dependent on the size of the target alphabet of the morphism. This makes it difficult, if not even impossible, to achieve a simple characterisation of those strings for which there exists an unambiguous erasing morphism with a certain finite target alphabet - in particular, a characterisation that is nearly as simple as in the case of nonerasing morphisms ( $c f$. Thm. 2.3). In order to obtain further results on the ambiguity of erasing morphisms, a profound knowledge of alphabet-specific ambiguity phenomena needs to be developed. 


\section{REFERENCES}

[1] C. Choffrut and J. Karhumäki, Combinatorics of words, edited by G. Rozenberg and A. Salomaa, Handbook of Formal Languages 1, Chap. 6. Springer (1997) 329-438.

[2] A. Ehrenfeucht and G. Rozenberg, Finding a homomorphism between two words is NPcomplete. Inform. Process. Lett. 9 (1979) 86-88.

[3] D.D. Freydenberger and D. Reidenbach, The unambiguity of segmented morphisms. In Proc. 11th International Conference on Developments in Language Theory, DLT $200 \%$. Lect. Notes Comput. Sci. (2007) 181-192.

[4] D.D. Freydenberger, D. Reidenbach and J.C. Schneider, Unambiguous morphic images of strings. Int. J. Found. Comput. Sci. 17 (2006) 601-628.

[5] M.R. Garey and D.S. Johnson, Computers and Intractability - A Guide to the Theory of NP-Completeness. W.H. Freeman and Co., New York (1979).

[6] T. Head, Fixed languages and the adult languages of OL schemes. Int. J. Comput. Math. 10 (1981) 103-107.

[7] T. Jiang, A. Salomaa, K. Salomaa and S. Yu, Decision problems for patterns. J. Comput. System Sci. 50 (1995) 53-63.

[8] A. Mateescu and A. Salomaa, Patterns, edited by G. Rozenberg and A. Salomaa, Handbook of Formal Languages 1, Chap. 4.6. Springer (1997) 230-242.

[9] D. Reidenbach, A non-learnable class of E-pattern languages. Theoret. Comput. Sci. 350 (2006) 91-102.

[10] D. Reidenbach, Discontinuities in pattern inference. Theoret. Comput. Sci. 397 (2008) 166-193.

[11] D. Reidenbach and J.C. Schneider, Morphically primitive words, in Proc. 6th International Conference on Words, WORDS 2007 (2007) 262-272.

[12] J.C. Schneider, Unambiguous erasing morphisms in free monoids, in Proc. SOFSEM 2009: Theorie and Practice of Computer Science. Lect. Notes Comput. Sci. 5404 (2009) 473-484.

Communicated by J. Berstel.

Received March 9, 2009. Accepted October 1st, 2009. 